\title{
Mães adolescentes em situação de rua: uma revisão sistemática da literatura
}

\author{
Homeless adolescent mothers: a systematic review of the literature
}

\author{
Anne Lise Sandoval Silveira Scappaticci ${ }^{1}$, Sergio Luis Blay ${ }^{2}$ \\ ${ }^{1}$ Doutora, Ciências da Saúde. Professor, Departamento de Psiquiatria, Universidade Federal de São Paulo - Escola Paulista de Medicina (UNIFESP-EPM), São Paulo, \\ SP. ${ }^{2}$ Doutor, Psiquiatria. Professor associado, Departamento de Psiquiatria, UNIFESP-EPM. \\ Este estudo foi realizado no Departamento de Psiquiatria da Universidade Federal de São Paulo - Escola Paulista de Medicina (UNIFESP-EPM), São Paulo, SP. Tese \\ apresentada 15 de março de 2006.
}

\section{Resumo}

Pouco se sabe sobre mães adolescentes que passam por gravidez ou maternidade fora de casa. Este estudo é uma revisão da literatura epidemiológica sobre esse fenômeno crescente. Os artigos foram identificados através de pesquisa nas seguintes bases de dados eletrônicas: MEDLINE, Lilacs, SciELO, PsychINFO, CINHAL, ERIC e Sociological Abstracts: 19 referências foram recuperadas. Os estudos mostram ampla heterogeneidade dos objetivos e dos métodos e revelam principalmente que as adolescentes têm elevadas taxas de abuso de substâncias, transtornos mentais, falta de apoio social, comportamento sexual, violência física e sexual, gravidez e interação mãe-criança problemáticas. Poucos artigos resultaram da revisão metodológica realizada sobre essa população. As mães adolescentes de rua têm grande exposição à violência, abuso de drogas e risco de problemas de saúde física e mental. Mais estudos centrados no estigma e em métodos de intervenção para esse grupo de mulheres são necessários nesta área, especialmente em populações culturalmente distintas.

Descritores: Adolescente de rua, gravidez na adolescência, maternidade.

\begin{abstract}
Little is known about mothers who are homeless during pregnancy and motherhood. This study is a review of the epidemiological literature about this increasing phenomenon. Articles were identified by searching the following electronic databases: MEDLINE, Lilacs, SciELO, PsychINFO, CINHAL, ERIC and Sociological Abstracts: 19 studies were retrieved. The studies showed a wide heterogeneity of objectives and methods, and mainly revealed that teenagers have high rates of substance abuse, mental disorders, lack of social support, sexual behavior, physical and sexual violence, troublesome pregnancy and mother-child interaction. Our methodological review found few articles about this population. Homeless adolescent mothers are extensively exposed to violence, drug abuse, and risk of physical and mental health problems. Further studies are needed about this topic, mainly involving culturally different populations, focusing on stigma and intervention methods for this group of women.
\end{abstract}

Keywords: Homeless adolescent, teenage pregnancy, motherhood.

Correspondência:

Anne Lise Scappaticci, Rua Dr. Diogo de Faria, 1337, Vila Clementino, CEP 04037-005, São Paulo, SP. E-mail: annelisescappaticci@yahoo.it

Apoio financeiro: Coordenação de Aperfeiçoamento de Pessoal de Nível Superior (CAPES)

Não há conflitos de interesse associados à publicação deste artigo.

Copyright (C) Revista de Psiquiatria do Rio Grande do Sul - APRS

Recebido em 15.03.09, aceito em 12.10.09. 


\section{Introdução}

A gravidez na adolescência tem sido foco da pesquisa em saúde. A literatura atual revela muito sobre os contextos de vida que levam adolescentes a fugir de casa: vitimização, violência doméstica, abuso sexual, abuso de substâncias, problemas de saúde mental, pobreza, relações sociais restritas e instabilidade residencial são frequentemente precursores da vida nas ruas entre os adolescentes ${ }^{1}$. Por outro lado, embora as famílias de adolescentes com filhos seja uma das subpopulações em situação de rua que mais cresce, relativamente poucos estudos têm focalizado a gravidez ou a paternidade entre adolescentes sem-teto ${ }^{2,3}$.

$\mathrm{O}$ fenômeno das famílias que vivem em situação de rua tem sido atribuído a vários fatores de risco interpessoais e psicológicos, incluindo doença mental ${ }^{4}$, históricos de abuso físico ou sexual ${ }^{5,6}$, violência doméstica e abuso de drogas 7 . Além disso, pesquisas sobre mães de baixa renda e sem-teto centradas nas condições sociais e na pobreza revelaram que as mães sem-teto tinham uma rede de apoio menor do que as mães que viviam em suas casas ${ }^{8}$. Existem também alguns estudos que indicam atrasos no desenvolvimento entre as crianças ${ }^{4,9,10}$ ou prejuízo no relacionamento mãe-filho devido ao abuso de drogas entre as mães que vivem nas ruas ${ }^{10}$.

Um amplo estudo sobre essa população foi realizado em Worcester (MA), EUA (Worcester Family Research Project, WFRP). Esse estudo epidemiológico recente investigou os fatores que podem proteger contra o fenômeno de famílias que vivem em situação de rua. Através do modelo de análise multivariada, detectou-se que os fatores de proteção incluíram receber subsídios para a habitação, ter ensino médio completo, ter mais pessoas em sua rede social e ter menos relações conflituosas. Fatores que reduziram o capital econômico e/ou social da família também estavam associados à falta de moradia. Por exemplo, internação para tratamento de problema de saúde mental nos últimos 2 anos e uso frequente de álcool ou heroína foram fatores de risco, apesar de serem raros na amostra ${ }^{11}$.

No Brasil, as famílias de adolescentes com filhos são uma grande preocupação ${ }^{12-14}$. No entanto, pelo que sabemos, não há estudos que resumam essa literatura sobre mulheres jovens que vivenciam a maternidade ou a gestação em abrigos temporários. $\mathrm{O}$ objetivo do presente estudo foi apresentar uma revisão de estudos epidemiológicos sobre gravidez e maternidade entre adolescentes que vivem em abrigos temporários. Especificamente, nossos objetivos foram: 1) consolidar os resultados disponíveis na literatura recente; e 2) identificar as principais características da saúde mental e dos comportamentos associados a esse fenômeno entre adolescentes.

\section{Métodos}

\section{Pesquisa da literatura}

\section{Fontes de pesquisa}

Os seguintes bancos de dados eletrônicos foram consultados: MEDLINE, Latin American Caribbean Literature in
Health Sciences Information (Lilacs), Scientific Electronic Library Online (SciELO), PsychInfo, CINHAL, ERIC and Sociological Abstracts. Além da pesquisa eletrônica, realizou-se também uma pesquisa manual de referências em artigos, livros, teses e trabalhos submetidos para publicação. Solicitou-se que investigadores enviassem separatas de estudos que não puderam ser encontrados e definiu-se 3 meses de prazo para o recebimento das informações.

Estratégia de busca em bancos de dados eletrônicos

Os critérios de inclusão foram deliberadamente amplos devido à dificuldade de encontrar estudos sobre o assunto. Como as mulheres que vivem em situação de rua estão muitas vezes associadas a abuso sexual e maus-tratos, doenças mentais, abuso de drogas, pobreza, assistência social, gravidez e originam-se de família desintegradas, inicialmente utilizou-se todas as palavras-chave associadas a esses temas, tais como: "adolescentes sem-teto" OU "crianças em acolhimento familiar" OU "maternidade na adolescência" OU "gravidez na adolescência” OU "relações mãe-filho".

\section{Seleção dos estudos epidemiológicos}

\section{Critérios de inclusão}

a) Todos os estudos eram artigos originais em inglês ou português. Decidiu-se centrar esta revisão na literatura recente, avaliando estudos realizados nos últimos 24 anos (19852009) quando o assunto tornou-se uma preocupação social.

b) Idade: com base nos critérios da Organização Mundial da Saúde (OMS) ${ }^{15}$, definiu-se adolescentes/jovens como tendo entre 10 e 19 anos. Estudos realizados com grandes amostras de adultos sem-teto, mas que incluíam adolescentes como um subgrupo, também foram incluídos.

c) Foram pesquisados estudos epidemiológicos com grandes amostras populacionais ou mesmo séries de casos em hospitais, ambulatórios ou centros de obstetrícia que incluíam adolescentes do sexo feminino, gravidez ou maternidade e adolescentes sem-teto vivendo em centros de acolhimento.

d) Uma descrição clara da metodologia do estudo era necessária, incluindo o delineamento do estudo, a estratégia de amostragem e os instrumentos de avaliação. Entrevistas presenciais, ou seja, questionários aplicados através de contato pessoal foram incluídas no estudo.

\section{Critérios de exclusão}

Foram excluídos artigos teóricos, investigações com uma descrição pouco clara ou pobre sobre a metodologia utilizada e manuscritos baseados em relatórios estatísticos anuais, tais como dados censitários e informações obtidas de forma indireta por meio de gráficos ou arquivos. Também foram excluídos estudos qualitativos, que serão abordados em outro estudo. Estudos envolvendo exclusivamente abuso de drogas, vírus da imunodeficiência humana (human immunodeficiency virus, HIV) e doenças sexualmente transmissíveis também foram excluídos. 


\section{Procedimentos}

A estratégia de pesquisa dos artigos foi a leitura dos títulos para encontrar artigos que investigassem mães adolescentes em situação de rua. Posteriormente, os resumos eram lidos e, em seguida, o artigo completo era lido. Quando o título e o resumo não deixavam claro se o estudo incluía um grupo de mães adolescentes, o texto integral era examinado.

Quando havia dúvida sobre a inclusão, o artigo era lido por um examinador independente e, então, a decisão de incluir ou excluir era tomada consensualmente por dois revisores independentes (AS, SLB). Os revisores não estavam cegos para os nomes dos autores, das instituições ou dos jornais e revistas.

\section{Avaliação da qualidade e gerenciamento de dados}

A qualidade metodológica dos estudos selecionados foi avaliada por dois revisores independentes (AS, SLB), que também extraíram os dados. Se os estudos não descreviam o tamanho da amostra de adolescentes, os autores dos artigos originais eram contatados para a obtenção de informações adicionais. O critério de qualidade foi definido como A ou B. Estudos de qualidade $\mathrm{A}$ foram pesquisas realizadas através de questionários válidos e confiáveis aplicados pessoalmente por entrevistadores treinados em amostras representativas do local (ou dos abrigos) selecionadas aleatoriamente, cujos sujeitos foram definidos como adolescentes que fugiram de casa. Estudos que não cumpriam um ou mais critérios de qualidade foram classificados como sendo de qualidade B.

\section{Coleta e análise dos dados}

Informações como país, ano de coleta de dados, medidas sociodemográficas, tamanho da amostra, taxas de prevalência e características metodológicas dos estudos (como delineamento, local, tipo de entrevista, definição de adolescente que fugiu de casa) foram obtidas dos trabalhos selecionados. Em primeiro lugar, os estudos foram analisados qualitativamente. Dependendo do tipo e do resultado dos estudos, uma meta-análise podia ser realizada.

\section{Resultados}

A estratégia de pesquisa resultou em 8.062 estudos. Os títulos dos artigos foram revisados de forma a excluir os que não eram de interesse para este projeto. Disso resultaram 3.682 artigos, cujos resumos foram lidos. Após excluir os artigos com base em seus resumos, examinou-se 278 manuscritos através de sua leitura na íntegra. Houve total concordância entre os examinadores. Um fluxograma do processo de seleção é apresentado na Tabela 1.

Tabela 1 - Fluxograma do processo de seleção dos artigos

\begin{tabular}{|c|c|c|c|c|c|c|}
\hline & $\begin{array}{l}\text { Título/ano de } \\
\text { publicação }\end{array}$ & $\begin{array}{c}\text { Autor/ } \\
\text { periódico }\end{array}$ & Objetivos & Amostragem & Delineamento & Principais achados \\
\hline 1 & $\begin{array}{l}\text { The impact } \\
\text { of continuing } \\
\text { illegal drug } \\
\text { use on teenage } \\
\text { pregnancy } \\
\text { outcomes } \\
\text { - a prospective } \\
\text { cohort study } \\
(2002)\end{array}$ & $\begin{array}{l}\text { Quinlivan \& } \\
\text { Evans } \\
\text { BJOG An } \\
\text { International } \\
\text { Journal of } \\
\text { Obstetrics and } \\
\text { Gynaecology }\end{array}$ & $\begin{array}{c}\text { Avaliar o impacto } \\
\text { do uso contínuo de } \\
\text { drogas ilegais nos } \\
\text { desfechos da gravidez } \\
\text { na adolescência. }\end{array}$ & $\begin{array}{c}456 \\
\text { adolescentes }\end{array}$ & $\begin{array}{l}\text { Estudo } \\
\text { de coorte } \\
\text { prospectivo e } \\
\text { multicêntrico }\end{array}$ & $\begin{array}{l}\text { Um aumento na incidência de } \\
\text { isolamento social, situação de rua e } \\
\text { violência doméstica foi observado } \\
\text { a partir de um ponto basal no } \\
\text { grupo que não usava drogas para } \\
\text { um pico no grupo multi-drogas. } \\
\text { A incidência de uma patologia } \\
\text { psiquiátrica identificada pelo DSM } \\
\text { foi significativamente maior no grupo } \\
\text { da maconha e no grupo multi-drogas. } \\
\text { As jovens grávidas usuárias de drogas } \\
\text { ilícitas eram mais propensas a fumar } \\
\text { cigarros e ingerir bebidas alcoólicas. } \\
\text { 40\% das participantes do estudo } \\
\text { deixaram de usar drogas ilegais } \\
\text { imediatamente antes ou durante a } \\
\text { gravidez. }\end{array}$ \\
\hline 2 & $\begin{array}{c}\text { Pregnancy } \\
\text { among three } \\
\text { national } \\
\text { samples of } \\
\text { runaway and } \\
\text { homeless youth } \\
\text { (1998) }\end{array}$ & $\begin{array}{l}\text { Greene \& } \\
\text { Ringwalt } \\
\text { Adolesc. } \\
\text { Health. }\end{array}$ & $\begin{array}{c}\text { Comparar as } \\
\text { estimativas da } \\
\text { prevalência de gravidez } \\
\text { entre jovens que } \\
\text { fugiram de casa e em } \\
\text { situação de rua em } \\
\text { diversos contextos. }\end{array}$ & $\begin{array}{c}\text { A análise } \\
\text { foi limitada } \\
\text { a } 1.988 \\
\text { adolescentes } \\
\text { do sexo } \\
\text { feminino a } \\
\text { partir de três } \\
\text { inquéritos. }\end{array}$ & $\begin{array}{l}\text { Técnicas de } \\
\text { amostragem } \\
\text { multiestágios }\end{array}$ & $\begin{array}{l}\text { As jovens mais velhas estavam } \\
\text { significativamente mais propensas } \\
\text { a ter engravidado do que as mais } \\
\text { jovens, as negras tinham mais } \\
\text { probabilidade do que as brancas. As } \\
\text { mulheres jovens que tinham estado } \\
\text { fora de casa por períodos mais longos } \\
\text { eram mais propensas a relatar já terem } \\
\text { engravidado. As jovens moradoras } \\
\text { de rua eram mais susceptíveis a ter } \\
\text { engravidado, seguidas por jovens que } \\
\text { viviam em abrigos e por jovens que } \\
\text { viviam com suas famílias. }\end{array}$ \\
\hline
\end{tabular}




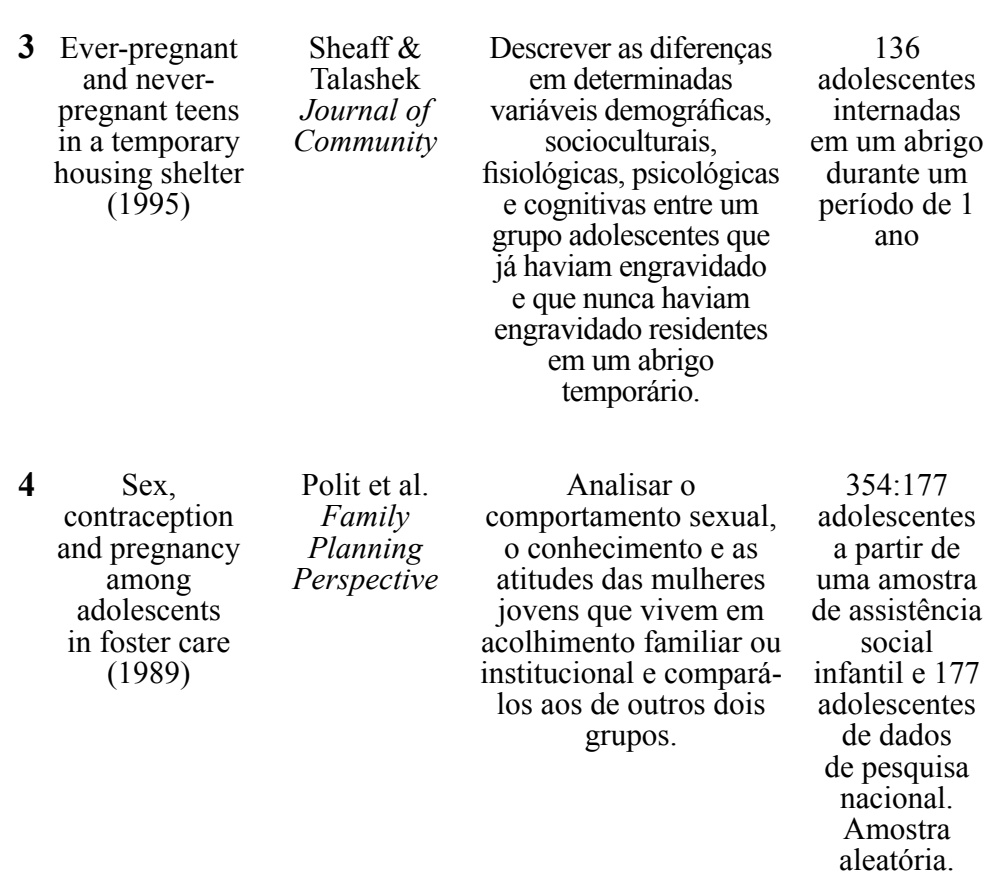

5 Pregnancy and childbirth: risk factors for homelessness? (1989)

6 Substance use and separation of homeless mothers from their children (2003)
Weitzman Family Planning Perspective
Explorar as relações entre situação de rua e dois fatores: gravidez e o número de filhos.
Zlontnick

et al.

Addictive

Behaviors
Examinar se as mães em situação de rua com problemas de uso de substâncias eram mais propensas a terem sido separadas dos filhos e se o uso recente de substâncias teve um impacto sobre a capacidade da família de receber a ajuda financeira do estado de maneira consistente ao longo do período de 15 meses de estudo (situação de custódia e abuso de substâncias).
Estudo retrospectivo, descritivo

Caso-controle (

(n) ter engravidado.

Quase metade da amostra que vivia em acolhimento familiar ou institucional afirmou que o sexo antes do casamento nunca é aceitável, ao contrário de $29 \%$ das adolescentes que viviam em casa. Elas também acreditam que uma gravidez não desejada antes do casamento deve ser resolvida dando-se o bebê para adoção. As adolescentes que viviam em casa eram mais propensas a ter beijado um menino, ter um namorado e ter tido uma relação sexual. Quase a metade das adolescentes que viviam em acolhimento familiar ou institucional e $38 \%$ daquelas que viviam em casa reconheceram que foram abusadas sexualmente. As adolescentes que viviam em casa eram mais sexualmente ativas do que as jovens que viviam em acolhimento familiar ou institucional. Comparando com o grupo da pesquisa nacional, as adolescentes que viviam em acolhimento familiar ou institucional tinham menos probabilidade de ter usado controle de natalidade na última relação sexual e a maioria era quase 2 anos mais jovem na primeira relação sexual.

704 famílias Caso-controle

Trinta e cinco por cento das mulheres em situação de rua estavam grávidas no momento da entrevista e $26 \%$ haviam tido filhos no ano anterior, comparadas com 6 e $11 \%$, respectivamente, de mulheres da amostra que vivia em casa. Além disso, ter um filho antes dos 18 anos estava significativamente relacionado à situação de rua, mas não ao tamanho da família.

e recebiam social.

104 mulheres que tiveram filhos com menos de 18 anos de idade.

Amostragem
por
conglomerados
multiestágios
com
estratificação

Apenas 29,1\% das mulheres tinham todos os filhos vivendo com elas durante todo o período de 15 meses de estudo. As mães que tinham sido separadas de seus filhos eram mais propensas a ter um transtorno de abuso de substâncias atual e de ter estado em situação de rua por pelo menos 1 ano em comparação com outras mães em situação de rua. 
7 The impact of childhood foster care and other out-home placement on homeless women and their children (1999)
Zlontnick

et al. Child

Abuse Neglect

Comparar as mulheres que tinham históricos infantis de acolhimento familiar ou outros tipos de acolhimentos com aquelas que não tinham tal histórico. em situação de rua

179 mulheres em situação de

rua.

$$
\begin{gathered}
\text { Amostragem } \\
\text { por } \\
\text { conglomerado } \\
\text { multiestágios } \\
\text { com } \\
\text { estratificação; } \\
\text { amostragem } \\
\text { aleatória }
\end{gathered}
$$

Estudo descritivo longitudinal. Grupo controle

\section{Examinar o vínculo materno-infantil em um grupo de pais adolescentes em situação de rua. \\ 8 Maternal- infant bonding in homeless adolescents and their infants

$\begin{array}{cc}\text { Rich } & \text { Examinar o vínculo } \\ \text { Maternal- } & \text { materno-infantil em } \\ \text { Child Nursing } & \text { um grupo de pais } \\ \text { Journal } & \begin{array}{c}\text { adolescentes em } \\ \text { situação de rua. }\end{array}\end{array}$
(1990)
9 Security of attachment and parenting: homeless and low-income housed mothers and infants (1999)

\section{Easterbrooks \& Graham American Journal of Orthopsychiatry}

Examinar a segurança do apego em bebês sem-teto e bebês de baixa renda que vivem em casa.

\section{7 pares mãe-filho que estavam participando do programa CAM Comparar os dados a uma amostra nacional - NCAST-scores $(\mathrm{n}=767)$.}

112 duplas mãe-bebê de famílias com baixos recursos econômicos: 55 duplas no grupo de sem-teto e 57 duplas no grupo que vive em casa.
10 Prevalence and correlates of survival sex among runaway and homeless youth (1999)
Greene et al. Examinar a prevalência American Journal of Public Health e correlacionar o sexo para sobrevivência entre jovens que fugiram de casa e em situação de rua.
631 adolescentes de abrigos para jovens e 528 jovens em situação de rua.
Caso-controle

Um terço das mulheres em situação de rua relataram terem sido criadas longe de seus pais.

Entre as mulheres menores de 18 anos de idade com filhos, a maioria $(61,5 \%)$ havia tido filhos que viveram em acolhimento familiar ou institucional. As mães em situação de rua, cujos filhos estavam vivendo em acolhimento familiar ou institucional, eram mais propensas a ter um filho em idade escolar, ter 35 anos de idade ou mais, ter um transtorno de uso de álcool ou drogas atual, ter sofrido abuso sexual infantil e ter fugido de casa (antes dos 18 anos).

A pontuação dos pais para o CAM não foi significativamente diferente da pontuação dos pais da amostra maior NCAST; este resultado sugere que as mães do CAM são sensíveis aos sinais infantis, respondem à angústia do bebê e se envolvem em comportamentos que promovem o crescimento emocional, cognitivo e social de seus filhos.

Apenas relatos de histórico de vitimização física ou sexual foram associados com a condição habitacional, com as mães sem-teto tendendo a relatar vitimização mais frequentemente do que as mães que moram em casa. Poucas diferenças foram encontradas entre as famílias que viviam em casa e aquelas semteto. Em vez de sugerir adaptação favorável por famílias sem-teto, porém, esses dados evidenciam as dificuldades enfrentadas por todas as famílias com filhos pequenos e baixos recursos econômicos.

Os dois grupos de crianças apresentaram baixa segurança de apego, enquanto que nenhuma relação foi encontrada entre a segurança de apego e a condição de habitação.

Caso-controle

Cerca de $28 \%$ das jovens de rua e $10 \%$ das jovens de abrigos relataram ter participado de sexo para sobrevivência, o que esteve associado a idade (16,1 anos para a amostra de abrigo e 18,1 anos para a amostra de rua), dias longe de casa (cerca de $46 \%$ para jovens que estiveram longe de casa por mais de 1 mês, em comparação com $78 \%$ dos jovens de rua), vitimização, comportamentos criminosos, uso de substâncias, tentativas de suicídio, doença sexualmente transmissível e gravidez 
11 Psychosocial aspects of Mexican-

American,

white, and

black teenage

pregnancy (Jul, 1987)
Felice et al. $J$ Adolesc

Health Care
Avaliar se adolescentes estadunidenses de origem mexicana grávidas têm características psicológicas diferentes de adolescentes brancas ou negras grávidas.
199

adolescentes estadunidenses de origem mexicana,

brancas e negras grávidas: 79

brancas, 76

estadunidenses de origem mexicana e 44 negras.
12 The association of foster care or kinship with adolescent sexual behavior and first pregnancy (Sept, 2001)
Carpenter et al.

Pediatrics
Investigar a relação entre viver em acolhimento por parentes ou em acolhimento institucional e comportamentos reprodutivos de alto risco em uma amostra nacionalmente representativa de mulheres.
13 Risk profile of homeless pregnant

adolescents and youth (Nov, 1991)

\section{Pennbridge et al. Journal of Adolescent \\ Health}

Examinar as necessidades de mulheres jovens grávidas em situação de rua, comparando os perfis de risco de jovens moradoras de rua grávidas e jovens grávidas que vivem com suas famílias.

\author{
Descrever as \\ características de \\ famílias em situação \\ de rua.
}

Estudo descritivo prospectivo
Dados de 9.620

mulheres:

acolhimento

familiar ou

institucional

$(\mathrm{n}=89)$,

acolhimento

por parentes

$(\mathrm{n}=513)$ e

comparação (n

$=9.018$ ).

Estudo transversal

Estudo grávidas em situação de rua e 85 jovens grávidas que vivem com suas famílias. transversal

\footnotetext{
14 Characteristics Bassuk et al. of sheltered homeless American Journal of families (1986)
}

80 mães em situação de rua e 151 crianças que viviam em 14 abrigos para famílias em Massachusetts.
.Adolescentes negras grávidas tinham maior probabilidade de estar na escola no momento da inscrição para o pré-natal $(\mathrm{p}<0,05)$. As adolescentes estadunidenses de origem mexicana eram mais propensas a se casar após a concepção e/ou parto e a amamentar seus bebês. Os pais das adolescentes estadunidenses de origem mexicana tinham mais probabilidade do que os outros pais de serem estudantes em tempo integral ou de estarem empregados. Apesar das adolescentes estadunidense de origem mexicana grávidas virem de famílias maiores, as adolescentes negras relataram um bom relacionamento mãe-filha com mais frequência. As adolescentes brancas grávidas relataram doença psiquiátrica na família com mais frequência.

$\mathrm{O}$ acolhimento familiar ou institucional foi associado com menor idade na primeira concepção e ter número de parceiros sexuais maior do que a mediana. $\mathrm{O}$ acolhimento por parentes

foi associado com menor idade na primeira relação sexual e na primeira concepção e ter número de parceiros sexuais maior do que a mediana.

Não houve diferenças entre o grupo de acolhimento por parentes e o grupo de acolhimento familiar ou institucional, mas os dois grupos diferiram significativamente do grupo de comparação.

A maioria das jovens vivia em casa e metade delas tinha sido encaminhada por um centro de detenção juvenil.

A maioria das gestantes jovens sem-teto é branca (41\%), e a maioria das jovens grávidas que viviam em casa é de origem hispânica (55\%). Em comparação, $58,7 \%$ das gestantes adolescentes sem-teto vêm de fora do país, com $35,2 \%$ de fora da Califórnia.

Aleatório

Apenas seis mães eram menores de 20 anos. Vinte e nove mulheres haviam estado envolvidas em pelo menos um relacionamento em que tinham sido agredidas, mais de dois terços dos casos de

violência relatados estavam relacionados ao álcool ou às drogas. Um terço das mães em situação de rua nunca haviam conhecido seus pais. Mais de dois terços descreveram, pelo menos, uma importante

ruptura familiar durante a infância. 
Mães adolescentes em situação de rua - ScAPPATICCI \& BLAY

15 The characteristics and needs of sheltered homeless and low-income housed mothers (1996)

$\begin{array}{ccc}\text { Bassuk et al. } & \text { Comparar as } & 220 \text { mães sem- } \\ \text { American } & \text { características de mães } & \text { teto vivendo } \\ \text { Medical } & \text { em situação de rua e } & \text { em abrigos e } \\ \text { Association } & \text { de baixa renda nos } & \text { 216 mães de } \\ & \text { domínios econômico, } & \text { baixa renda } \\ & \text { psicossocial e de saúde } & \text { vivendo em } \\ & \text { física. } & \text { suas casas (sem } \\ & & \text { experiência de } \\ & \text { situação de rua) } \\ & & \text { e recebendo } \\ & \text { assistência } \\ & & \text { social. }\end{array}$

16 Determinants of behavior in homeless and lowincome housed preschool children (1997)
Ellen et al.

Pediatrics

Descrever as características de escolar sem-teto e de baixa renda e identificar determinantes ambientais e familiares do seu comportamento. crianças em idade
17 Prevalence of mental health and substance use disorders among

homeless and low-income housed mothers (1988)
Ellen et al. $A m J$ Psychiatry
Comparar a prevalência dos transtornos do

DSM-III-R entre mães sem-teto e de baixa renda vivendo em casa com a prevalência desses transtornos entre todas as mulheres na Pesquisa Nacional de Comorbidades.
220 mães semteto e 216 mães que vivem em casa recebendo assistência

social.
Caso-controle

77 mães semteto vivendo em abrigos e 90 mães de baixa renda vivendo em suas casas com filhos em idade préescolar $(\mathrm{n}=167$ pré-escolares) foram avaliadas.

Caso-controle não-pareado
Caso-controle não-pareado

As mães sem-teto e as mães que viviam em casa tiveram taxas similares de transtornos psiquiátricos e causados por substâncias.

Os dois grupos apresentaram maiores taxas atuais e ao longo da vida de depressão e abuso de substâncias do que todas as mulheres da Pesquisa

Nacional de Comorbidades. Os dois grupos também apresentaram altas taxas de transtorno de estresse pós-traumático e duas ou mais doenças crônicas. A prevalência ao longo da vida de transtornos relacionados ao álcool entre as mães de baixa renda, no entanto, foi quase o dobro, e outras formas de abuso de drogas foram quase três vezes maior do que doenças comparáveis na Pesquisa Nacional de Comorbidades. 
18 Determinants of health and service use patterns in homeless and low-income housed children (1988)

\section{Weinreb et al. Pediatrics}

Descrever os problemas de saúde e o uso de serviços de saúde em crianças que vivem em suas casas.

\author{
sem-teto e 334 \\ crianças de \\ baixa renda \\ vivendo em \\ casa.
}

293 crianças

Caso-controle crianças sem-teto experimentaram um número maior de sintomas de doença aguda, como febre, infecção de ouvido, diarréia e asma. As consultas médicas em serviços de emergência e ambulatoriais foram mais frequentes no grupo sem-teto. O sofrimento emocional das mães esteve associado de maneira independente a sintomas da doença aguda e uso frequente de serviços ambulatoriais e de pronto-socorro.

19 Sexual practices and attitudes of street youth in Belo Horizonte, Brazil (1993)

\section{Raffaelli et al. Soc.Sci.Med.}

\section{Apresentar informações sobre sexualidade e comportamento de risco entre jovens de rua do} Brasil (HIV).
291 meninos e 88 meninas (estudo piloto com 65 jovens)
Selecionadas segundo critérios de estudo específicos.

Essas criancas e adolescentes envolvem-se em um comportamento sexual que os coloca em risco de adquirir doenças sexualmente transmissíveis, incluindo HIV/ AIDS, e reforçam que o sexo é um comportamento multi-determinado e bem estabelecido nessa população.

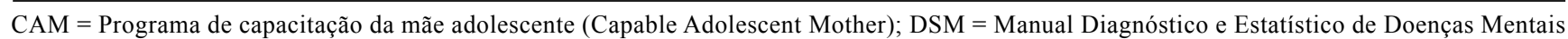
(Diagnostic and Statistical Manual of Mental Disorders); HIV = vírus da imunodeficiência humana (human immunodeficiency virus); NCAST-scores

= Escala de enfermagem para a avaliação da interação mãe-bebê durante a amamentação (Nursing Child Assessment Feeding Scale);

Finalmente, 19 artigos satisfizeram os critérios de seleção e foram incluídos nesta revisão (Tabela 2). Esses artigos foram todos classificados como sendo de qualidade B de acordo com os critérios adotados.

\begin{tabular}{lc}
\hline \multicolumn{2}{c}{ Tabela 2 - Fluxograma do processo de seleção } \\
\hline Artigos & $\mathbf{n}$ \\
Artigos potenciais identificados & 29.537 \\
Artigos excluídos devido ao título & 26.779 \\
Artigos excluídos devido ao resumo & 2.585 \\
Resumos avaliados & 2.758 \\
Artigos excluídos por não preencherem os critérios de inclusão & 132 \\
Artigos avaliados em relação à metodologia & 173 \\
Artigos incluídos nesta revisão & 41 \\
\hline
\end{tabular}

O WFRP, que foi um grande estudo sobre famílias de baixa renda e em situação de rua realizado em Worcester, EUA, durante a década de 1990, produziu uma grande quantidade de dados. Os artigos baseados nesse estudo, ${ }^{9,16}$ foram incluídos nesta revisão. Com exceção de um estudo realizado na Austrália ${ }^{7}$ e outro no Brasil ${ }^{17}$, todas as pesquisas foram realizadas nos EUA.

\section{Avaliação metodológica}

Os estudos foram realizados com diferentes enfoques metodológicos. As características dos delineamentos dos estudos são descritas a seguir.

\section{Objetivos}

Os estudos tiveram vários objetivos. Houve investigações sobre: 1) drogas na gravidez e na maternidade ${ }^{7,10}$; 2) gravidez e seu impacto ${ }^{18-21}$; 3) interação mãe-filho ${ }^{9,23}$; 4) aspectos psicossociais de mulheres sem-teto $\left.{ }^{4,6,8,11,20,24} ; 5\right)$ o comportamento sexual de risco entre jovens sem-teto ou que vivem em abri$\left.\operatorname{gos}^{17,21,26} ; 6\right)$ viver em situação de rua e saúde mental ${ }^{11,16,27}$.

\section{Contextos}

As investigações foram realizadas em diferentes contextos: 1) amostras populacionais ${ }^{9,16,17,21}$; 2) estudos em hospital e estudos clínicos ${ }^{7,24,27} ; 3$ ) estudos em abrigos ${ }^{9,10,18,19,21-23}$.

\section{Idade dos sujeitos}

No que diz respeito às idades dos sujeitos, sete estudos incluíram exclusivamente adolescentes entre 7 e 19 anos de idade $^{7-17,22-24,27}$, e o restante teve as adolescentes como um subgrupo de uma amostra maior de mães adultas ${ }^{4,9,16,19-20,26}$.

\section{Principais características de saúde mental e comportamento}

Devido à heterogeneidade dos objetivos de cada estudo, os resultados foram agrupados de acordo com as principais áreas de investigação: abuso de substâncias, transtornos mentais, falta de apoio social, comportamento sexual, violência física e sexual, gravidez e interação mãe-filho. 


\section{Abuso de substâncias}

Muitos estudos consideraram o abuso de substâncias como sendo um dos principais fatores de risco para a população de rua, seja como um desfecho principal $^{7,10}$ seja como um achado paralelo aos principais objetivos do estudo ${ }^{4-26}$. Os dois estudos nos quais o abuso de drogas foi a principal preocupação ${ }^{7,10}$ apresentaram seus resultados dentro de um contexto mais amplo. Quinlivan e Evans ${ }^{7}$ correlacionaram um grupo de sujeitos não usuários de drogas com um grupo usuário de várias drogas com incidência significativamente maior de isolamento social, situação de rua e violência doméstica. Eles descobriram especificamente que os adolescentes usuários de maconha eram significativamente mais propensos a ser socialmente isolados, viver em situação de rua ou ser vítima de violência doméstica do que aqueles no grupo de não usuários. Zlotnick et al. ${ }^{10}$ consideraram o grande impacto do uso de substâncias sobre a vida das mulheres e concluíram que a utilização apenas nos últimos 30 dias contribuiu para a perda da custódia dos filhos. As mães que fizeram uso de drogas por períodos mais longos não apresentaram o desfecho esperado pelos autores. Outros fatores parecem influenciar a manutenção da relação da mãe com seu filho, como a duração do período em que permaneceram sem-teto: as mães que não perderam a guarda dos filhos permaneceram sem-teto por períodos mais curtos. A condição de ser sem-teto também trouxe outros problemas que podem ser considerados como negligência parental, tais como o fato da criança não frequentar a escola. Os autores concluíram que houve uma relação circular entre estabilidade familiar, pobreza e renda: "a perda da estabilidade familiar está associada à perda da custódia dos filhos (guarda legal); quando há perda da guarda dos filhos, a renda pode ser reduzida; e a perda de rendimento desestabiliza ainda mais a integridade da família". Em ambos os estudos, o uso de drogas ilícitas foi associado ao aumento da incidência de uso de álcool simultaneamente.

\section{Transtornos mentais}

Os transtornos mentais são um problema de saúde geralmente descrito em mães adolescentes que vivem fora de casa nesses estudos. Os instrumentos de avaliação utilizados para avaliar problemas de saúde mental foram: a Entrevista Clínica Estruturada para o DSM (Manual Diagnóstico e Estatístico das Doenças Mentais) edição para não pacientes (SCID-NP - Structure Clinical Interview for DSM disorders), a Entrevista Clínica Estruturada para o DSM-III-R, versão para não pacientes (DSM-III-R), a Escala de Avaliação de Sintomas (SCL-90 - Symptom Checklist-90-Revised) e a Escala de Depressão do Centro de Estudos Epidemiológicos (CES-D Center for Epidemiologic Studies Depression Scale). Alguns pesquisadores não explicaram como as suas avaliações dos transtornos mentais foram realizadas. Pennbridge et al. ${ }^{24}$ revelaram que a amostra de sem-teto de seu estudo apresentou vários perfis de saúde mental, especialmente em relação a tentativas de suicídio e depressão (30\%). Da mesma forma, os sujeitos foram duas vezes mais propensos a serem diagnosticados com um grave distúrbio mental. Em uma investigação do vínculo mãe-filho comparando mães sem-teto e de baixa renda, Easterbrooks \& Graham ${ }^{9}$ não encontraram associação entre saúde mental e condição habitacional. Os problemas mentais estavam presentes nos dois grupos do estudo de forma equitativa. Da amostra total, dois terços ( $\mathrm{n}=$ 74) das mães relataram sintomas depressivos atuais graves o suficiente para serem classificados dentro da faixa clínica. Em um estudo sobre a prevalência de saúde mental e transtornos por uso de substâncias entre mães sem-teto e mães de baixa renda que viviam em suas casas, Weinreb et al. ${ }^{26}$ descobriram que ambos os grupos apresentaram taxas elevadas de depressão maior: $45 \%$ para o grupo de sem-teto e $42,8 \%$ para o grupo de baixa renda que vivia em casa. Os grupos também apresentaram transtorno de estresse pós-traumático (TEPT), 36,1 e 34,1\%, respectivamente.

\section{Saúde mental de mães e filhos}

A saúde mental da mulher é tema dos estudos de Bassuk

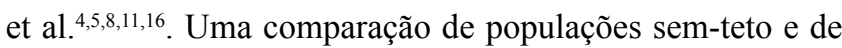
baixa renda mostrou que o estado emocional da mãe, além de vários estressores, prevê fortemente o desfecho negativo de uma criança para as duas escalas de comportamento infantil (Child Behavior Checklists - CBCL).

\section{Falta de apoio social}

Quase todos os estudos enfatizaram a vulnerabilidade de adolescentes grávidas ou mães, a vulnerabilidade da população sem-teto e a necessidade de programas de assistência inovadores para essa população. Por exemplo, comparando-se adolescentes sem-teto com jovens de baixa renda que viviam com suas famílias ${ }^{4,16,26}$, foi salientado que tanto as famílias sem-teto quanto as de baixa renda alojadas tinham redes de apoio fragmentadas, enquanto os indivíduos sem-teto eram significativamente mais isolados socialmente do que os indivíduos alojados. $\mathrm{O}$ estudo de Bassuk et al. ${ }^{8}$ apontou a fragilidade econômica da população sem-teto e sua instabilidade habitacional. Pennbridge et al. ${ }^{24}$ argumentaram que as adolescentes grávidas e sem-teto não somente corriam maior risco de uma grande variedade de problemas, mas também tinham muito menos apoio social do que seus pares que viviam em casa. O principal achado de um estudo realizado por $\operatorname{Rich}^{23}$ foi de que não havia diferenças significativas entre as adolescentes que viviam em abrigos sob os cuidados de

\footnotetext{
* N.T. Todas as ocorrências de citações diretas de textos originalmente em inglês foram traduzidas livremente com base no texto fornecido pelos autores do artigo.
} 
um programa assistencial para mães adolescentes em Nova Jersey e as adolescentes da população em geral, ressaltando, assim, a importância do apoio social em abrigos para mulheres com o intuito de nutrir uma relação entre a jovem mãe e seu filho. Baseando-se em entrevistas, Bassuk et al. ${ }^{4}$ constataram que quase dois terços de sua amostra não tinha ou tinha poucas relações de apoio e um quarto da amostra mencionou os filhos como o principal apoio. Esse estudo correlacionou a falta de apoio social para a família: a maioria dos famílias eram chefiadas por mulheres; a maioria tinha históricos de instabilidade residencial; e a maioria recebeu auxílio para famílias com filhos dependentes. A busca por habitação havia levado as famílias entrevistadas a se mudarem 6,6 vezes durante os 5 anos anteriores ao primeiro episódio de situação de rua. Durante os 5 anos anteriores, $85 \%$ tinham vivido em alojamentos super povoados e mais de $50 \%$ haviam morado em alojamentos emergenciais.

\section{Comportamento sexual}

Confirmando as informações da literatura ${ }^{29}$, os autores dos estudos analisados ${ }^{17,18}$ consideraram que as adolescentes semteto estavam mais expostas a comportamentos de alto risco, como uso de drogas, gravidez precoce ${ }^{19}$, sexo para sobrevivên$\mathrm{cia}^{24}$ e ausência de controle de natalidade ${ }^{6,17}$. Comparando as adolescentes que viviam em suas próprias casas com aquelas que viviam em lares adotivos, Polit et al. ${ }^{6}$ concluíram que as adolescentes que viviam em casa estavam mais propensas a ter relações sexuais e eram mais sexualmente ativas do que as que viviam em lares adotivos. Um grande número de adolescentes que viviam em acolhimento familiar ou institucional tinham sido abusadas, e se a adolescente já havia tido relações sexuais voluntariamente, isso estava positiva e fortemente relacionado ao número de lares de acolhimento em que havia vivido. As adolescentes em acolhimento familiar ou institucional estavam menos propensas a ter usado um método contraceptivo durante a sua primeira relação sexual e estavam substancialmente menos propensas a tê-lo usado no episódio mais recente de relação sexual (45 versus $64 \%$ ). Análises comparando usuários de assistência social e a amostra nacional sugeriram que as adolescentes que recebiam assistência social infantil não apenas correm mais risco de ter relações sexuais antes do casamento do que seus pares, mas também são menos informadas sobre controle da natalidade e sexualidade humana.

Estar em situação de rua e a duração dessa situação, juntamente com episódios anteriores de gravidez ${ }^{22}$, apareceram nesses estudos como fatores de risco para outra gravidez. Preocupações em relação ao abuso sexual ${ }^{18} \mathrm{e}$ famílias não-intactas também foram manifestadas por esses autores. As adolescentes em situação de rua estavam mais envolvidas com prostituição ou sexo para sobrevivência, apresentaram pior perfil de saúde mental e mais problemas médicos, relataram porcentagem muito maior de experiências de abuso e tinham tido sua primeira experiência sexual mais cedo do que seus pares que não viviam em situação de rua ${ }^{24}$. Além disso, em cada categoria de droga, de drogas intravenosas a cigarros, as jovens grávidas sem-teto relataram mais uso de drogas do que as adolescentes que viviam em suas casas. Em um estudo sobre adolescentes sem-teto em uma grande cidade brasileira, Raffaelli et al. ${ }^{17}$ salientaram que as respostas revelaram que as adolescentes que viviam nas ruas estavam em um ambiente sexual arriscado.

\section{Violência física e sexual}

Não encontramos nenhum estudo que investigou a violência e as mães adolescentes sem-teto como seu principal objetivo. Os estudos analisados abrangeram um vasto leque de temas, e quando os autores investigaram os termos "abuso" ou "maus tratos", o tipo ou a gravidade do abuso vivenciado pelos entrevistados ou as relações entre os agressores e as vítimas não estavam claros. Ao descrever suas amostras, a maioria dos estudos revelou que mulheres adultas e adolescentes de baixa renda e sem-teto eram vítimas de violência física ou abuso sexu$\mathrm{a}^{1,16,18,20,22}$. Easterbrooks \& Graham ${ }^{9}$ afirmaram que as mulheres sem-teto relataram mais vitimização física ou sexual do que as mães que viviam em suas casas. Comparando grupos de adolescentes que já haviam e nunca haviam estado grávidas, Sheaff $\&$ Talashek ${ }^{22}$ demonstraram que o estupro (abuso sexual por uma pessoa desconhecida) foi relatado por $20 \%$ do grupo que tinha estado grávida, contra $7 \%$ daquelas que nunca estiveram grávidas. Em geral, eles descobriram que havia altos níveis de violência, com mais de $40 \%(n=136)$ relatando agressão física. Polit et al..$^{6}$ afirmaram que seu estudo confirmou uma tendência das vítimas de abuso sexual na infância demonstrarem maior risco de comportamento sexual precoce, um fator presumivelmente responsável por pelo menos alguns dos riscos elevados entre os usuários de assistência social infantil. Investigando a violência física e sexual em grupos de mães sem-teto que viviam em abrigos e mães de baixa renda que viviam em suas casas, Bassuk et al. ${ }^{8}$ concluíram que, para a maioria das entrevistadas, os episódios de abuso físico e sexual e agressão tiveram início antes da idade adulta ( $40 \%)$. Durante a vida adulta, quase dois terços de toda a amostra sofreu agressão física grave por parceiro íntimo. Os estudos analisados confirmam a tendência demonstrada pela literatura sobre a população de adolescentes ou adultos sem-teto ${ }^{28}$ : a violência doméstica esteve significativamente associada ao uso de drogas e gravidez.

\section{Gravidez}

Os estudos mencionados aqui ${ }^{17-19,22}$ parecem concordar sobre o fato de que a idade e a duração da situação de rua foram fatores-chave para a previsão de gravidez. Greene \& Ringwalt ${ }^{18}$ concluíram que "as jovens mais velhas estavam significativamente mais propensas a ter engravidado do que as mais jovens, as negras tinham mais probabilidade do que as brancas. As mulheres jovens que tinham vivido fora de casa por períodos mais longos eram mais propensas a ter engravidado. As jovens moradoras de rua foram as mais susceptíveis a ter engravidado, seguidas por jovens que viviam 
em abrigos e por jovens que viviam com suas famílias". Sheaff \& Talashek ${ }^{22}$ acrescentaram que as adolescentes que já haviam engravidado apresentavam: "significativamente mais históricos de estupro e atividade sexual voluntária do que as adolescentes que nunca haviam engravidado" e concluíram: "As adolescentes desta amostra relataram o dobro de gravidezes do que as adolescentes da população geral. Elas também têm poucos recursos aos quais recorrer quando a gravidez ocorre: limitados recursos familiares, educacionais e de habilidades cognitivas para resolver os problemas". Comparando 704 famílias sem-teto que recebiam assistência social em Nova Iorque com 524 famílias que viviam em suas casas, Weitzman ${ }^{19}$ afirmou que $26 \%$ das mulheres sem-teto entrevistadas tinham tido filhos no ano anterior, comparadas a 6 e 11\% das mulheres que viviam em casa. Outro achado interessante que emerge desses estudos é a gravidez como uma forma de proteção para a adolescente e para o bebê. As adolescentes que participaram do programa Capable Adolescent Mothers foram as que decidiram criar seus bebês, que demonstraram disponibilidade para cuidar de si mesmas e que não estavam usando drogas ou álcool ${ }^{23}$. Essa associação entre gravidez e a opção de deixar a rua e o abuso de drogas também foi encontrada por Quinlivan \& Evans ${ }^{7}$, que relataram que $40 \%$ (181) das participantes de seu estudo deixaram de usar drogas ilegais imediatamente antes ou durante a gravidez precoce. Tem-se sugerido que os fatores motivadores para uma adolescente ter um bebê também a motivam a parar de usar drogas ilícitas.

\section{Interação mãe-filho}

Os resultados do estudo realizado por Bassuk et al. ${ }^{8}$ envolvendo populações sem-teto e de baixa renda foram surpreendentes: as crianças sem-teto e de baixa renda vivenciaram adversidades significativas em suas vidas, sendo que as crianças em situação de rua enfrentaram mais estresse. Além de vários estressores, o estado emocional da mãe previu efetivamente o desfecho negativo do filho nas duas subescalas CBCL. Os dois grupos tinham redes de apoio fragmentadas, com os indivíduos sem-teto significativamente mais socialmente isolados do que aqueles que viviam em suas casas. Weinreb et al. ${ }^{26}$ examinaram os determinantes de saúde e os padrões de uso de serviços entre 627 crianças sem-teto e de baixa renda que viviam em suas casas. Além dos achados relatados anteriormente, como o fato de que as crianças em situação de rua serem significativamente mais jovens e mais propensas a ter mudado de endereço no ano anterior, as mães das crianças sem-teto estavam mais propensas a relatar que seus filhos tinham estado de saúde regular ou ruim e tinham maior frequência de consultas ambulatoriais e de atendimento de emergência em comparação com os seus pares que viviam em casa. Easterbrooks \& Graham ${ }^{9}$ relataram que tanto as crianças sem-teto quanto as que viviam em casas de famílias com baixos recursos econômicos corriam risco de comportamento de apego inseguro. Esses autores sugeriram que as manifestações de insegurança ambivalente podem ser uma maneira da criança de lidar com a disponibilidade materna inconsistente. Para as crianças que vivem em ambientes caóticos, super povoados ou imprevisíveis (incluindo abrigos para sem-teto e alojamentos informais para mães), isso pode ser um comportamento adaptativo de certa forma. Nesse sentido, Rich $^{23}$ descobriu que as adolescentes podiam interagir adequadamente com seus filhos em um contexto que oferecesse continuidade. Como Rich ${ }^{23}$, Zlotnick et al. ${ }^{20}$ enfatizaram que a interação mãe-filho pode ser problemática em abrigos para famílias, onde a privacidade é rara; o impacto dessa variável sobre a interação mãe-filho não foi suficientemente estudado por nenhum dos autores. Zlotnick et al. ${ }^{20}$ concluíram que a situação de rua ameaçava a integridade da família. Eles enfatizaram que os programas para famílias sem-teto precisavam abordar esses problemas nas suas intervenções, bem como considerar os desafios impostos pelos ambientes dos abrigos para sem-teto, onde as ações e os diálogos dos pais eram constantemente presenciados por outros pais, pelas crianças e pelos funcionários do abrigo.

\section{Discussão}

Como o presente estudo tratou de um fenômeno atual, buscou-se realizar uma revisão sistemática de estudos epidemiológicos a respeito do tema das mães sem-teto ou das adolescentes grávidas. A busca resultou em 19 artigos. Nem todos abordaram diretamente o problema, embora tenham contribuído para a nossa compreensão do fenômeno, revelando facetas e características dessa população que, quando vistas em sua complexidade contextual, pareciam interligadas: abuso de substâncias, saúde mental, falta de apoio social, violência física e sexual, gravidez, interação mãe-filho e comportamento sexual.

Esses dados indicam que muitas das jovens que chefiavam essas famílias sem-teto agora têm dificuldade em estabelecerem-se como adultas autônomas e empregadas; elas continuarão dependentes das instituições a longo prazo para enfrentar sua maternidade sem sistemas de apoio adequados.

\section{Principais características de saúde mental e comportamento}

Há estudos que abordam a questão da resiliência, em que a população sem-teto sobrevive em condições de pobreza, com menos recursos econômicos e apoios sociais do que outros segmentos da população. A falta de apoio familiar e a violência presentes em todas as amostras foram reconhecidas como um substrato da situação de rua da família. Um grande número das mulheres estudadas haviam sido agredidas física ou sexualmente durante a vida. Assim, não é surpreendente que o TEPT, os transtornos do uso de substâncias e a depressão maior tenham sido desproporcionalmente elevados, com taxas de TEPT três vezes maiores do que na população em geral do sexo feminino ${ }^{16}$. 
Um ponto que aparece nas discussões na literatura é que a gravidez, o abuso de drogas e outros padrões de comportamento parecem ser estratégias para a superação de um contexto familiar caótico. Por exemplo, Quinlivan \& Evans ${ }^{7}$ argumentaram que "em alguns casos, o uso de drogas é um mecanismo de fuga para uma adolescente que é confrontada com um ambiente com falta de apoio e abusivo". Easterbrooks et al. ${ }^{9}$ salientaram que "para crianças que vivem em ambientes caóticos, super povoados ou imprevisíveis (incluindo abrigos para sem-teto e alojamentos informais nas casas de outras pessoas), as manifestações de insegurança ambivalente podem ser uma maneira da criança de lidar com a situação."

Alguns estudos indicam que adolescentes sem-teto que vivem em abrigos temporários foram abusadas sexualmente ou fisicamente, ou ambos ${ }^{22}$. Greene e Ringwalt ${ }^{18}$ argumentaram que o fenômeno do abuso sexual pode ser um fator-chave, juntamente com o estupro e as dificuldades econômicas enfrentadas pela população sem-teto, que leva esses indivíduos ao uso de drogas, à prática do sexo para sobrevivência e a outros padrões de comportamento de risco. A ligação entre a violência (estupro) e o comportamento sexual de adolescentes sem-teto foi destacada em um estudo realizado por Raffaelli et al., ${ }^{17}$ no qual os autores demonstraram que o estupro é um meio coercitivo para o estabelecimento de hierarquia em gangues de rua, bem como uma forma de violência, especialmente em relação às mulheres. Apesar de o estudo de Raffaelli et al. ${ }^{17}$ fazer parte de um projeto sobre soroprevalência do HIV e fatores de risco que realizou intervenções para reduzir o risco de infecção pelo HIV entre crianças e adolescentes que viviam ou trabalhavam nas ruas de Belo Horizonte, a quarta maior cidade do Brasil, o estudo apresenta informações sobre sexualidade e comportamento de alto risco entre os adolescentes que vivem nas ruas. Este estudo incluiu um estudo piloto que investigou gravidez e contracepção, o qual está diretamente relacionado com a presente revisão.

Outros estudos que abrangem o uso de drogas ${ }^{7,10}$ foram incluídos nesta revisão, de acordo com os mesmos critérios, considerando que eles não apenas trataram o abuso de substâncias, mas trouxeram à luz aspectos importantes para se compreender as adolescentes grávidas e mães adolescentes sem-teto. A maioria dos estudos apontou a necessidade de reunir essas mães em abrigos, contando com equipes preparadas para receber a mãe e a criança e com políticas sociais que possam proporcionar apoio e atenuar o problema. Esta situação de abrigo temporário parece ser necessária por um período de tempo durante o qual contribui para a formação de um vínculo entre as jovens mães e seus filhos. Vários autores que trabalharam com essas populações enfatizaram essa preocupação $0^{30,31}$. Rich ${ }^{32}$ mencionou uma menina em sua amostra, apontando que: "Rubin afirma que uma mulher pode dar á luz uma criança, mas ela não se torna mãe sozinha. Ela precisa de um sistema de apoio social para ajudá-la a realizar a tarefa da maternidade". Esse autor também apontou a dificuldade dessas meninas em aceitar a autoridade dos funcionários dos abrigos e sua propensão para fugir da situação ${ }^{32}$. Em um estudo que comparou os resultados e a relação custo-efetividade entre clínicas de atendimento pré-natal para adolescentes com enfoque interdisciplinar abrangente e serviços obstétricos tradicionais para adultos, Bensussen-Walls e Saewyc ${ }^{31}$ constataram que as pacientes de clínicas para adolescentes faltavam menos às consultas e estavam mais propensas a ter acompanhamento de longo prazo e a amamentar seus bebês se comparadas àquelas das clínicas para adultos. Os custos foram menores para os desfechos das clínicas para adolescentes, enquanto que os custos relativos a trabalho de parto prematuro foram semelhantes.

\section{Avaliação metodológica}

Conforme mencionado na seção Resultados, verificouse que todos os estudos foram classificados como B no que diz respeito à qualidade da metodologia utilizada. Além disso, observou-se que houve grande heterogeneidade em relação aos métodos utilizados. As investigações tiveram objetivos, contextos e agrupamentos por idade diferentes. Os instrumentos de avaliação nem sempre foram mencionados nem se indicou como foram administrados ou quais eram as qualificações dos pesquisadores que aplicaram os questionários. No caso da violência doméstica, os investigadores não especificaram se o sujeito era a vítima ou o agressor. Da mesma forma, ao considerar a violência, os estudos muitas vezes não deixaram claro se o foco era a violência física ou sexual. Além disso, se essa informação de fato estava presente, o estudo não esclarecia se $o$ abuso ocorreu durante a infância ou na idade adulta. Assim, embora os estudos confirmem a correlação reconhecida na literatura entre populações pobres sem-teto, violência e transtornos mentais, a generalização sobre essas questões é difícil. A grande heterogeneidade metodológica entre os estudos limitaram as possibilidades de análise quantitativa dos resultados. Devido a esses resultados, as investigações nesse campo seriam muito beneficiadas se os grupos interessados nesse tema pudessem padronizar os instrumentos de avaliação utilizados.

\section{Áreas que necessitam de mais pesquisas}

Esta revisão revelou diversas áreas que necessitam de mais pesquisas. Não encontramos nenhum estudo, considerando os efeitos que um abrigo ou outro contexto de situação de rua tem sobre o desenvolvimento infantil e a paternidade e as características das interações entre pais e filho. Temas como o estigma associado a ser um adolescente sem-teto com filhos, as políticas públicas, os estudos longitudinais, os desfechos da gravidez, a maternidade e o desenvolvimento infantil permanecem obscuros e deveriam ser objeto de futuras investigações.

\section{Conclusão}

Nossa revisão encontrou poucos estudos epidemiológicos enfocando o tema. Além disso, essas investigações foram realizadas utilizando-se diferentes estruturas metodológicas, 
o que impediu um melhor agrupamento de dados. Os dados coletados indicam que adolescentes grávidas ou mães adolescentes que vivem nas ruas têm altas taxas de problemas comportamentais e mentais e alta exposição a situações de violência. Além disso, os dados evidenciam os riscos potenciais desse contexto de vida nas ruas ou em abrigos sobre a organização da relação entre a mãe adolescente e seu filho.

\section{Limitações do estudo}

Esta revisão tem diversas limitações. Em primeiro lugar, estudos focados principalmente em áreas afins, tais como abuso de drogas, depressão, depressão na gravidez ou em outras amostras populacionais podem não ter sido incluídos nesta revisão. Isso pode ter acontecido se a presença de adolescentes grávidas ou mães sem-teto não foi relatada nos métodos ou resultados do estudo ou se um estudo centrou-se exclusivamente sobre um tema sem tocar nas vicissitudes da gravidez na adolescência ou das mães adolescentes sem-teto. A segunda preocupação diz respeito ao viés de publicação. Estudos publicados em revistas não indexadas não foram incluídos nesta revisão. A maioria dos estudos publicados foi realizada no EUA; portanto, comparações interculturais não foram abordadas. Finalmente, devido às características estigmatizada do problema e às dificuldades de amostragem, os dados podem revelar uma visão parcial da questão. Em suma, uma vez que esta é uma população em crescimento, são necessários mais estudos para investigar a interação complexa entre vida, saúde e situação de rua das mães adolescentes.

\section{Referências}

1. Whitbeck LB, Hoyt DR, Ackley KA. Families of homeless and runaway adolescents: a comparison of parent/caretaker and adolescent perspectives on parenting, family violence, and adolescent conduct. Child Abuse Negl. 1997;21(6):517-28.

2. National Coalition for the Homeless. Homeless families with children. NCH Fact Sheet 1995 [cited 2009 Feb 12]; Available from: http://www.nationalhomeless. org/publications/facts/families.pdf

3. US Conference of Mayors. Hunger and homelessness survey: a status support on hunger and homelessness in America's cities. Hunger and Homelessness Survey 2004 [cited 2009 Feb 12]; Available from: http://www.usmayors.org/uscm/hungersurvey/2004/onlinereport/HungerAndHomelessnessReport2004.pdf

4. Bassuk EL, Rubin L, Lauriat AS. Characteristics of sheltered homeless families. Am J Public Health. 1986;76(9):1097-101.

5. Bassuk EL, Buckner JC, Perloff JN, Bassuk SS. Prevalence of mental health and substance use disorders among homeless and low-income housed mothers. American J of Psychiatry 1998;155:1561-4.

6. Polit DF, Morton TD, White CM. Sex, contraception and pregnancy among adolescents in foster care. Fam Plann Perspect. 1989;21(5):203-8.

7. Quinlivan JA, Evans SF. The impact of continuing illegal drug use on teenage pregnancy outcomes--a prospective cohort study. BJOG. 2002;109(10):1148-53.
8. Bassuk EL, Weinreb LF, Buckner JC, Browne A, Salomon A, Bassuk SS. The characteristics and needs of sheltered homeless and low-income housed mothers. JAMA. 1996;276(8):640-6.

9. Easterbrooks MA, Graham CA. Security of attachment and parenting: homeless and low-income housed mothers and infants. Am J Orthopsychiatry. 1999;69(3):337-46.

10. Zlotnick C, Robertson MJ, Tam T. Substance use and separation of homeless mothers from their children. Addict Behav. 2003;28(8):1373-83.

11. Bassuk EL, Weinreb LF, Dawson R, Perloff JN, Buckner JC. Determinants of behavior in homeless and low-income housed preschool children. Pediatrics. 1997;100(1):92-100.

12. Gama SGN, Szwarcwald CL, Leal MC. Experiência de gravidez na adolescência Fatores associados e resultados perinatais entre puérperas de baixa renda. Cad Saúde Pública. 2002,18(1):153-61.

13. Ribeiro ERO, Barbieri MA, Bettiol H, Silva AAM. comparação entre dois coortes de mães adolescentes em município do Sudeste do Brasil. Rev Saúde Pública. 200;34(2):136-42.

14. Freitas GVS, Botega NJ. Gravidez na adolescência. Prevalência de depressão, ansiedade e ideação suicida. Rev Assoc Med Bras. 2002;48(3):245-9.

15. WHO. Organization Mundial de La Salud. Necessidades de la salud de los adolescents. Ginebra: OMS; 1977.

16. Bassuk EL, Buckner JC, Perloff JN, Bassuk SS. Prevalence of mental health and substance use disorders among homeless and low-income housed mothers. Am J Psychiatry. 1998;155(11):1561-4.

17. Raffaelli M, Campos R, Merritt AP, et al. Sexual practices and attitudes of street youth in Belo Horizonte, Brazil. Street Youth Study Group. Soc Sci Med. 1993;37(5):661-70.

18. Greene JM, Ringwalt CL. Pregnancy among three national samples of runaway and homeless youth. J Adolesc Health. 1998;23(6):370-7.

19. Weitzman BC. Pregnancy and childbirth: risk factors for homelessness? Fam Plann Perspect. 1989;21(4):175-8.

20. Zlotnick C, Robertson MJ, Wright MA. The impact of childhood foster care and other out-of-home placement on homeless women and their children. Child Abuse Negl. 1999;23(11):1057-68.

21. Greene JM, Ennett ST, Ringwalt CL. Prevalence and correlates of survival sex among runaway and homeless youth. Am J Public Health. 1999.

22. Sheaff L, Talashek M. Ever-pregnant and never-pregnant teens in a temporary housing shelter. J Community Health Nurs. 1995;12(1):33-45.

23. Rich OJ. Maternal-infant bonding in homeless adolescents and their infants. Matern Child Nurs J. 1990;19(3):195-210.

24. Pennbridge J, Mackenzie RG, Swofford A. Risk profile of homeless pregnant adolescents and youth. J Adolesc Health. 1991;12(7):534-8.

25. Carpenter SC, Clyman RB, Davidson AJ, Steiner JF. The association of foster care or kinship care with adolescent sexual behavior and first pregnancy. Pediatrics. 2001;108(3):E46.

26. Weinreb L, Goldberg R, Perloff J. Health characteristics and medical service use patterns of sheltered homeless and low-income housed mothers. J Gen Intern Med. 1998;13(6):389-97.

27. Felice ME, Shragg GP, James M, Hollingsworth DR. Psychosocial aspects of Mexican-American, white, and black teenage pregnancy. J Adolesc Health Care. 1987;8(4):330-5.

28. Goodman L. The prevalence of abuse among homeless and housed poor mothers: a comparison study. Am J Orthopsychiatry. 1991;61(4):489-99.

29. Kidd SA, Scrimenti K. Evaluating child and youth homelessness. Eval Rev. 2004;28(4):325-41.

30. Garrett SC, Tidwell R. Differences between adolescent mothers and nonmothers an interview study. Adolescence. 1999;34(133):91-105.

31. Bensussen-Walls W, Saewyc EM. Teen-focused care versus adult-focused care for the high-risk pregnant adolescent: an outcomes evaluation. Public Health Nurs. 2001;18(6):424-35

32. Rich OJ. Vulnerability of homeless pregnant and parenting adolescents. J Perinat Neonatal Nurs. 1992;6(3):37-46.Referências 